\title{
Fiber-based Diffuser Sheet for Liquid Crystal Display Backlight Unit
}

\author{
Taehyung Kim ${ }^{1,2}$, Eun Soo Lee ${ }^{1}$, Won Young Jeong', Dae Young Lim¹, and Suk-Won Choi ${ }^{2}$ * \\ ${ }^{1}$ Department of Textile Convergence of Biotechnology \& Nanotechnology, \\ Korea Institute of Industrial Technology, Ansan 426-791, Korea \\ ${ }^{2}$ Department of Advanced Materials Engineering for Information and Electronics, Kyung Hee University, \\ Yongin 446-701, Korea
}

(Received March 18, 2014 : revised April 17, 2014 : accepted April 17, 2014)

\begin{abstract}
A fiber diffuser sheet based on poly (ethylene naphthalate) (PEN) and poly (methylpentene) (PMP) has shown potential for liquid crystal display backlight units, but these materials have an interfacial adhesion problem. To improve the interfacial adhesion between the fibers and matrix components, we have proposed the use of amorphous poly (cyclohexane-1,4-dimethylene terephthalate) (Tritan) instead of PEN. Furthermore, the fabrication processes have been optimized and simplified to improve the optical and mechanical properties of the sheet. As a result, the most effective fiber content for achieving the best haze characteristics of a sample consisting of Tritan and PMP has been identified.
\end{abstract}

Keywords : Fiber, Matrix, Diffuser, Liquid crystal display, Backlight unit

OCIS codes : (160.2290) Fiber materials; (230.1980) Diffusers; (290.0290) Scattering

\section{INTRODUCTION}

The liquid crystal display (LCD) is one of the most widely employed flat-panel displays in multimedia devices such as mobile phones, computer monitors, televisions, etc. [1]. In the field of LCDs, many researchers have been making strong efforts to develop backlight units (BLUs) with good optical properties, namely, high luminance, good luminance uniformity, and a wide viewing angle [2-5]. In the BLUs, a diffuser sheet that can provide uniform brightness by spreading the light is a key component. Although diffuser sheets have been developed extensively, most research has been on bead-type sheets [5-7]. Recently, new optical diffuser sheets based on fiber technology have been studied, and their potential has been demonstrated [8]. However, detailed studies on diffuser sheets based on fiber technology have not yet been conducted.

In a previous work, poly (ethylene naphthalate) (PEN) and poly (methylpentene) (PMP) were chosen for the fiber and matrix components, respectively, in such a fiber-based diffuser sheet [8]. Although they showed potential, these materials exhibited an interfacial adhesion problem. As the PEN content was increased, the diffuser sheet crumbled easily. Thus, no optimum ratio of PEN to PMP could be found. In this study, amorphous poly (cyclohexane-1,4dimethylene terephthalate) (Tritan) was used as the fiber component, which led to improved interfacial adhesion between the matrix and fibers. Then, various fiber contents were examined to find the composition leading to the most effective haze and total transmittance characteristics of this diffuser system.

\section{PREVIOUS DIFFUSER SHEET LACKING INTERFACIAL ADHESION}

In the previous films [8], fabricated by islands-in-the-sea (INS) conjugate melt-spinning [9], the PEN and PMP $\left(\right.$ TPX $^{\circledR}$ RT18, Mitsui Chemical) showed vulnerability to crumpling, depending on the PEN content. In particular, as the PEN content was increased, the diffuser sheet crumbled easily. Figure 1(a) shows a diffuser sheet made from PEN and PMP by INS conjugate melt-spinning. After the diffuser sheet was crumpled into a ball, it broke into fragments, as shown in Fig. 1(b). To analyze the cause of this phenomenon, we measured the tensile stress-strain curves of a sheet sample consisting of pure PMP and a sample with 40 vol\% PEN diffuser. The samples were cut into $2.54 \times$

\footnotetext{
*Corresponding author: schoi@khu.ac.kr

Color versions of one or more of the figures in this paper are available online.
} 


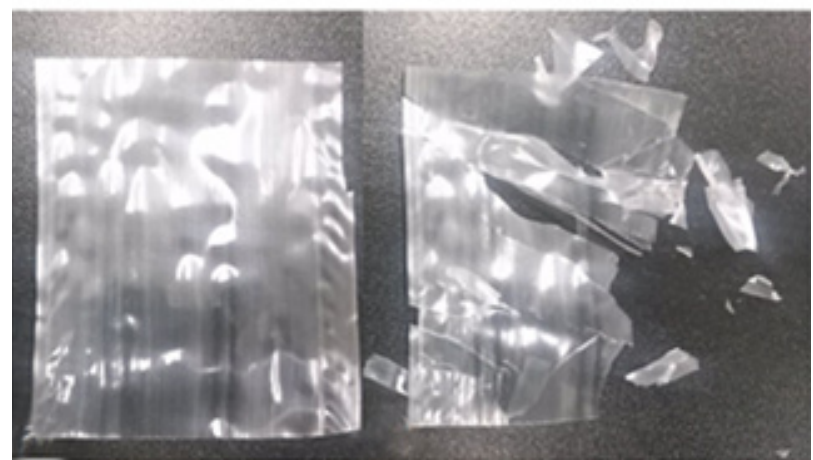

(a)

(b)

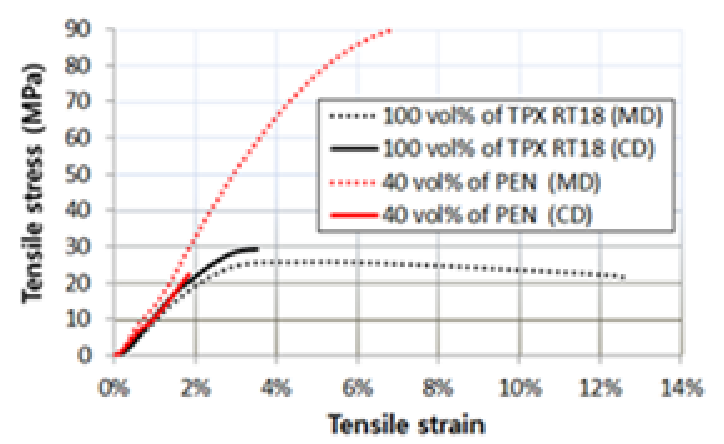

(c)

FIG. 1. Diffuser sheet made of the PEN and PMP $\left(\right.$ TPX $^{\circledR}$ RT18) (a) before and (b) after it was crumpled into a ball. (c) Tensile stress-strain curves of sheets made from pure PMP $\left(\mathrm{TPX}^{\circledR}\right.$ RT18) and 40 vol\% PEN. MD and CD indicate, respectively, the machine direction and cross direction.

$100.0 \mathrm{~mm}$ strips and preconditioned at $75 \%$ relative humidity and $25^{\circ} \mathrm{C}$ for $48 \mathrm{~h}$. The tensile properties were measured on an Instron tester (Norwood, MA, USA) according to the ASTM D882 standard. The initial grip separation was set to $50 \mathrm{~mm}$, and the cross-head speed was set to $50 \mathrm{~mm} / \mathrm{min}$ with a load cell of $50 \mathrm{kgf}$. At least five identical specimens were measured per experiment. Increasing the PEN fiber content to $40 \mathrm{vol} \%$ PEN led to more brittle fracture and lower strains of failure in both the machine direction (MD) and cross direction (CD), as compared to those for pure PMP. The poor durability of the 40 vol\% PEN sample can be fully explained by the tensile properties shown in Fig. 1(c).

\section{SHEET FABRICATION}

In order to fabricate the proposed diffuser sheet with improved interfacial adhesion, amorphous Tritan (Tritan ${ }^{\mathrm{TM}}$ copolyester TX2001, Eastman) and PMP were used as the fiber and matrix, respectively. Each material was obtained in pellet form and then melted and extruded by a singlescrew extruder with temperature profiles of $240^{\circ} \mathrm{C}-277^{\circ} \mathrm{C}$ and $200^{\circ} \mathrm{C}-255^{\circ} \mathrm{C}$. Under the pressure in the extruder, the
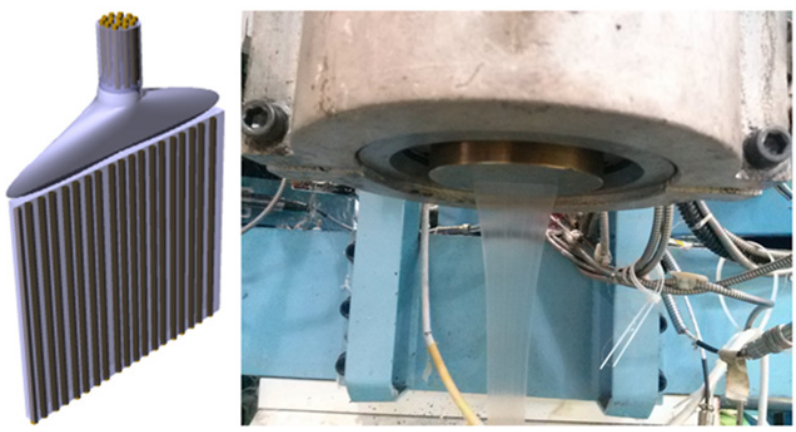

FIG. 2. Schematic illustration of the INS conjugate spinning setup and photograph of the real spinning setup.

melted fiber component passes through a spin pack with 3808 holes and then comes out of the slit-die, which creates the INS film consisting of a large number of individual islands of one polymer surrounded by a sea or matrix of another polymer. Unlike the conventional INS conjugate melt-spinning process, in which the sea polymer has to be removed by dissolving it with a solvent or by melting, a simple INS conjugate melt-spinning process without the dissolution step was focused on here, thereby reducing the number of complex work processes. The resulting film was then wound around the winding roll machine. The respective speeds of the casting roll, cooling roll, and take-off unit were 3,4 , and $6.4 \mathrm{~m} / \mathrm{min}$. A schematic illustration of the INS conjugate spinning setup and a photograph of the real spinning setup are shown in Fig. 2. In contrast to earlier approaches, in this approach a slit-die was utilized instead of a T-die to increase the fiber packing. In addition, the additional stretching process to match the birefringence of the fibers with that of the matrix was not adopted, for convenience of the manufacturing process [8], since our focus here was on a simple INS conjugate meltspinning process.

\section{RESULTS AND DISCUSSION}

Although we did not adopt an additional stretching process, the fabricated diffuser sheet still showed optically isotropic and birefringent characteristics, because some of the elongation necessary to achieve good birefringence was achieved in the winding process. In general, a crossed polarizer condition is utilized to confirm birefringence. Figure 3(a) shows the optical isotropy of the pure PMP (TPX $^{\circledR}$ RT18) sample. On the other hand, Fig. 3(b) shows the birefringence of the sample with a $30 \mathrm{vol} \%$ Tritan fiber content. According to the Jones matrix method, the transmittance of a sample placed at an azimuth angle $\Psi$ with respect to the transmission axes of the crossed polarizers will behave as follows [10]: 


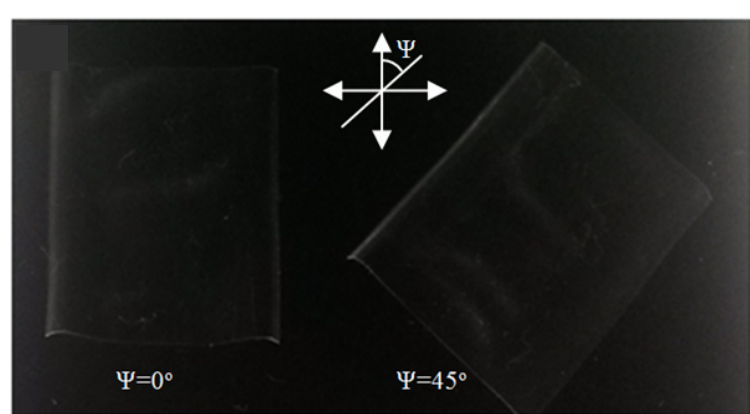

(a)

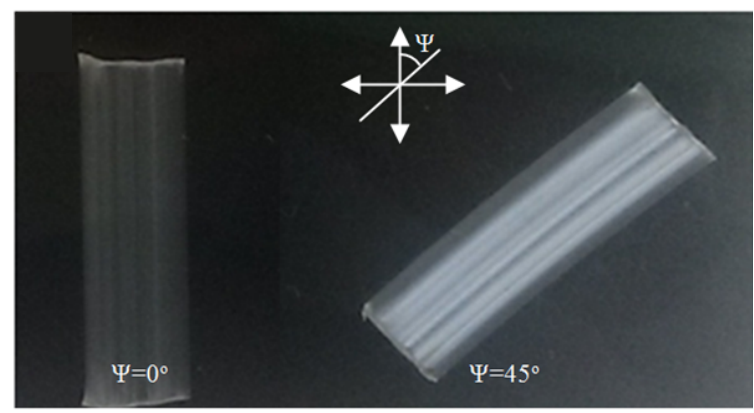

(b)

FIG. 3. Fabricated samples: (a) isotropy of the sample with 100 vol\% PMP and (b) birefringence of the sample with 30 vol $\%$ Tritan. The arrows indicate the directions of the polarizers.

$M=\left[\begin{array}{ll}1 & 0 \\ 0 & 0\end{array}\right]\left[\begin{array}{cc}e^{-i \Gamma / 2} \cos ^{2} \Psi+e^{i \Gamma / 2} \sin ^{2} \Psi & -i \sin (\Gamma / 2) \sin (2 \Psi) \\ -i \sin (\Gamma / 2) \sin (2 \Psi) & e^{i \Gamma / 2} \cos ^{2} \Psi+e^{-i \Gamma / 2} \sin ^{2} \Psi\end{array}\right]\left[\begin{array}{ll}0 & 0 \\ 0 & 1\end{array}\right]$

The obtained transmittance for $\Psi=45^{\circ}$ is then given as

$T=\frac{1}{2} \sin ^{2} \frac{\Gamma}{2}=\frac{1}{2} \sin ^{2}\left[\frac{\pi\left(n_{e}-n_{o}\right) d}{\lambda}\right]$

where $\Gamma$ is the phase retardation, $n_{e}-n_{o}$ is the birefringence, and $d$ is the thickness. When $\Psi=45^{\circ}$, maximum brightness is achieved, whereas $T=0$ when $\Psi=0^{\circ}$ or $90^{\circ}$. Therefore, an angle of $\Psi=45^{\circ}$ was used to obtain the bright state in Fig. 3(b).

Figure 4 shows the cross-sectional view of the fabricated diffuser sheets based on Tritan fiber and the PMP matrix. The morphology was observed by using a scanning electron microscope (SEM) (SU810, Hitachi, Japan). An image analysis technique was used to quantify the characteristic sizes of the fibers and their distributions in the proposed diffuser systems.

We fabricated nine kinds of diffuser sheets with different fiber contents (10-90 vol\% Tritan) by INS conjugate meltspinning to find the content yielding the best haze and total transmittance characteristics for this diffuser system. As seen in Fig. 4, the characteristic sizes of the fibers became larger as the fiber content increased. For $10 \mathrm{vol} \%$ fiber, fibers of $5 \mu \mathrm{m}$ in diameter are observed occasionally.

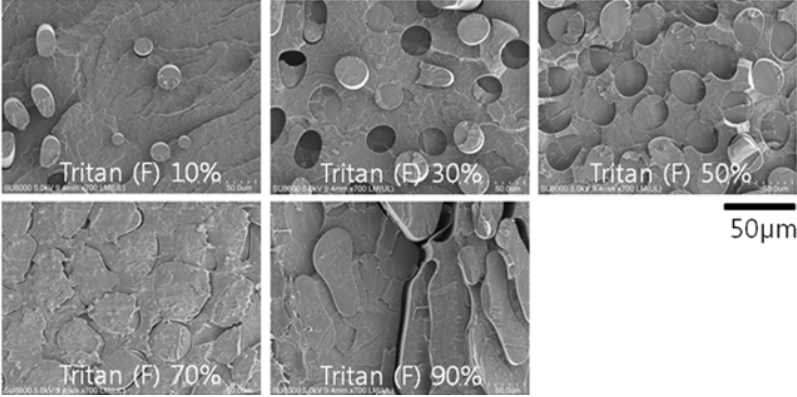

FIG. 4. Scanning electron micrographs of the proposed diffuser sheets consisting of different amounts of Tritan and PMP.

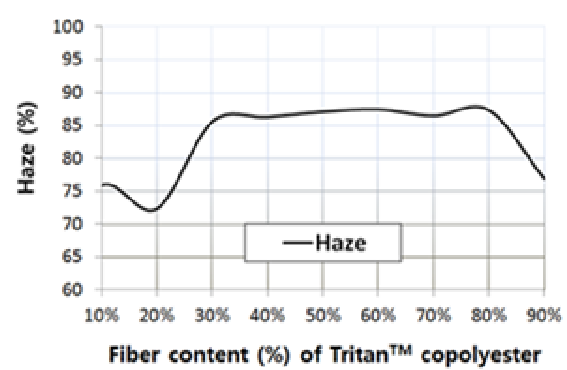

(a)

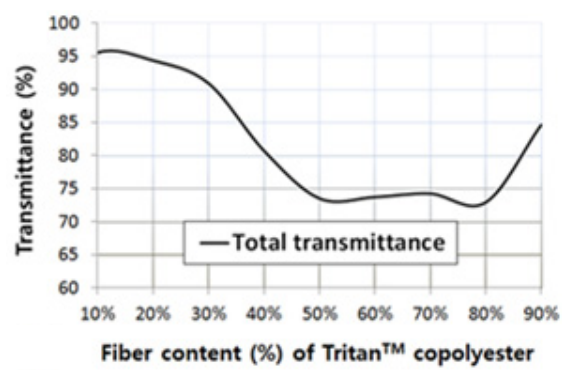

(b)

FIG. 5. (a) Haze and total transmittance characteristics for different fiber contents.

At $50 \mathrm{vol} \%$ fiber content, fiber diameters of $20 \mu \mathrm{m}$ are mainly observed. In addition, as the fiber content became larger, the fibers became closer in the matrix, until agglomeration occurred at $70 \mathrm{vol} \%$ fiber content because of the larger, closer fibers. These characteristic sizes and distributions of the fibers obviously affected the optical properties of the obtained sheets.

Figure 5(a) shows the haze characteristics for different fiber contents. The haze was large, above $85.5 \%$, for 30 $80 \mathrm{vol} \%$ fiber content values. Contrary to expectations, the haze values did not continuously increase with fiber content. Above $30 \mathrm{vol} \%$, the haze values were nearly saturated, and a maximum of $87.4 \%$ was reached at 60 vol\%. Figure 5(b) shows the dependence of the total transmittance values on the Tritan fiber content. Although the haze and total transmittance do not always exhibit opposite behavior, they tend to do so in our case: high haze values were associated with low transmittance, and 
vice versa. When the fiber content was $80-90$ vol $\%$, the total transmittance unexpectedly increased. In this region, the dispersed fibers assembled with each other in the matrices, and large domains composed of the fiber materials were obtained. Hence, the total transmittance was increased owing to the decrease in scattering between the fibers and matrix components. Consequently, the haze characteristics were also decreased. Thus, taken together, the haze and transmittance both had reasonably high values at a 30 vol\% fiber content, of $85.5 \%$ and $90.9 \%$, respectively, in this case.

Figure 6 shows the tensile stress-strain curves of the

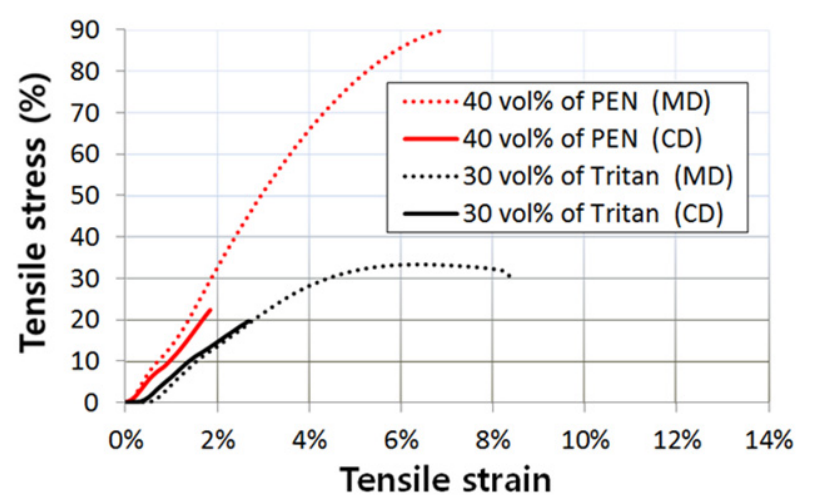

FIG. 6. Tensile stress-strain curves of diffuser sheets with 40 vol\% PEN and 30 vol\% Tritan.

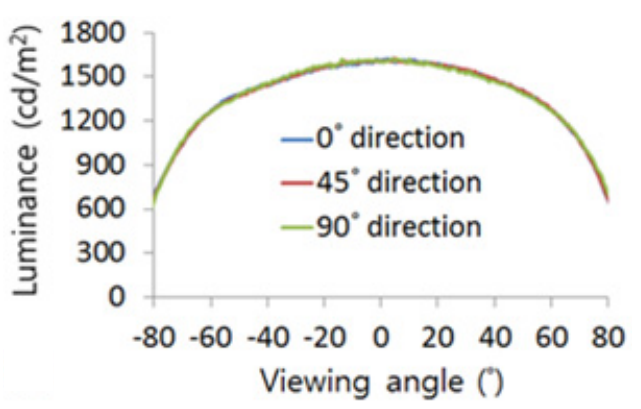

(a)

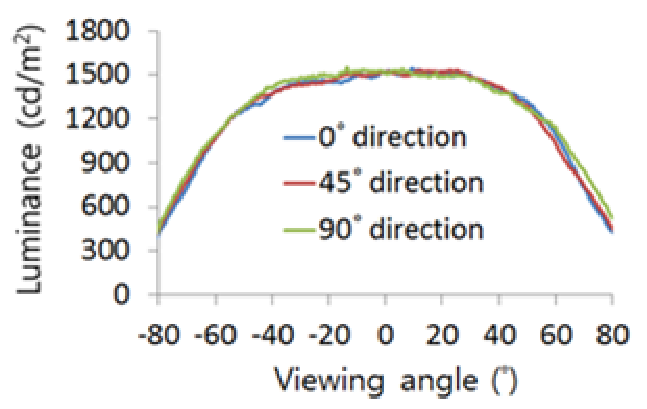

(b)

FIG. 7. Angular distributions of the luminance with respect to the angles along the horizontal $\left(0^{\circ}\right)$, diagonal $\left(45^{\circ}\right)$, and vertical $\left(90^{\circ}\right)$ directions of the BLUs with (a) a commercially available diffuser sheet and with (b) our fiber-based diffuser sheet fabricated in this work. diffuser PMP sheets with 40 vol\% PEN and 30 vol\% Tritan. The use of $30 \mathrm{vol} \%$ Tritan fiber led to more ductile fracture and increased strain of failure in both the MD and $\mathrm{CD}$, as compared to those obtained with $40 \mathrm{vol} \%$ PEN. The improved durability performance of the sample with 30 vol\% Tritan can be fully explained by the tensile results in Fig. 6. The PMP and PEN used previously were semicrystalline thermoplastic polymers, and it is known that combinations of semicrystalline polymers lead to interfacial adhesion problems between the fibers and matrix component. On the other hand, Tritan was well known to be an amorphous copolymer. Thus, from Fig. 6, it was confirmed that the use of amorphous Tritan instead of semicrystalline PEN effectively improved the interfacial adhesion.

Figure 7 shows angular distributions of the luminance with respect to the angles along the horizontal $\left(0^{\circ}\right)$, diagonal $\left(45^{\circ}\right)$, and vertical $\left(90^{\circ}\right)$ directions of the BLUs with a commercially available diffuser sheet and the fiber-based diffuser sheet fabricated in our study. The angular distributions of the luminance with respect to the angles were measured by using the EZContrast XL88 system (ELDIM). As shown in Fig. 7, the brightness profile of the BLU with our diffuser sheet was comparable with that of the BLU with the conventional diffuser sheet.

\section{CONCLUSION}

In a previous work, we proposed a diffuser sheet based on fiber technology and confirmed the feasibility of this approach. In this work, we made various attempts to improve the optical and mechanical properties of this diffuser system. To improve the interfacial adhesion, amorphous PET copolymer was proposed as the fiber component. To simplify the complex fabrication processes, the dissolution step and additional stretching process were omitted. In addition, a slit-die was utilized instead of a T-die to increase the fiber packing. Then, the most effective fiber content for maximizing the optical performance was identified for samples consisting of amorphous Tritan fibers and a PMP matrix.

\section{ACKNOWLEDGMENT}

The authors would like to express thanks to Yeo Hoon Yoon, Hyun Kyung Lee, Jae Kyoung Lee, Yoon Jin Kim, Joo Hwan Yoo, Dr. Jung Nam Im (Korea Institute of Industrial Technology), and Prof. Moo-Sung Lee (Chonnam National University) for their helpful advices. This research was supported by a grant from the Fundamental R\&D Program for Core Technology of materials (10037182, Structuring Technologies for Multi- functionally Converged Nanofibers) funded by Ministry of Trade, Industry \& Energy, Republic of Korea. This work was also partially supported by the National Research Foundation of Korea 
grant funded by the Ministry of Science, ICT \& Future Planning (Grant 2012R1A2A2A06046931).

\section{REFERENCES}

1. S. R. Park, O. J. Kwon, D. Shin, S. H. Song, H.-S. Lee, and H. Y. Choi, "Grating micro-dot patterned light guide plates for LED backlights," Opt. Express 15, $2888-2899$ (2007).

2. S. T. Hur, S.-W. Choi, and M. Lee, "An edge-lit backlight unit for small portable liquid crystal displays with a newly designed single pyramid sheet," J. Korean Phys. Soc. 58, 392-395 (2011).

3. S. T. Hur, S.-W. Choi, and M. Lee, "A direct-lit backlight unit for a 32-inch liquid crystal display incorporating a newly designed pyramid sheet," J. Korean Phys. Soc. 60, 1196-1198 (2012).

4. J. S. Seo, T. E. Yeom, and J. H. Ko, "Experimental and simulation study of the optical performances of a wide grid polarizer as a luminance enhancement film for LCD backlight applications,” J. Opt. Soc. Korea 16, 151-156 (2012).

5. J. H. Kwon, H. J. Jun, J. S. Gwag, and H. S. Lee, "Effect of wavelength-dependent scattering on the color chromaticity of the LCD backlight," J. Opt. Soc. Korea 17, 275-278 (2013).

6. G. Kim, "A PMMA composite as an optical diffuser in a liquid crystal display backlighting unit (BLU)," Eur. Polym. J. 41, 1729-1737 (2005).

7. G. Park, T. S. Aum, J. H. Kwon, J. H. Park, B. K. Kim, and J. K. Shin, "Characterization and modeling light scattering in diffuser sheets," J. Korean Phys. Soc. 54, 44-48 (2009).

8. T. Kim, S. Kim, D. Y. Lim, and S.-W. Choi, "A novel diffuser sheet comprising nanosized birefringent fibers embedded within an isotropic polymer matrix," Opt. Commun. 295, 125-128 (2013).

9. K. Yoon, B. Hsiao, and B. Chu, "Functional nanofibers for environmental applications," J. Mater. Chem. 18, 5326-5334 (2008).

10. P. Yeh and C. Cu, Optics of Liquid Crystal Displays, 2nd ed. (Wiley, New York, USA, 2009), p. 173. 